\title{
Combined Fourier and Zero Crossing Technique for Frequency Measurement in Power Networks in the Presence of Harmonics
}

\author{
Milenko B. Đurić, Željko R. Đurišić \\ Faculty of Electrical Engineering \\ University of Belgrade \\ Bul. kralja Aleksandra 73, 11000 Belgrade, Serbia and Montenegro \\ Phone: +381113218340, e-mail: mdjuric@etf.bg.ac.yu, djurisic@etf.bg.ac.yu
}

\begin{abstract}
A new approach to the design of a digital algorithm for network frequency estimation is proposed. The algorithm is derived using Fourier and zero crossing technique. The Fourier method is used as digital filter and zero crosing technique is applied to the cosine and sine components of the original signal which can be corrupted by higher harmonics. The algorithm showed a very high level of robustness as well as high measurement accuracy over a wide range of frequency changes and it can be used for frequncy tracking in power networks when the higher harmonics are presented in voltage or current signals. The theoretical basis and practical implementation of the technique are discribed. To demonstrate the performance of the developed algorithm, computer simulated, as well as real life data records, are processed.
\end{abstract}

\section{Key words}

Frequncy measurement, computer relaying, fourier method, zero crossing technique

\section{Introduction}

The frequency of a power network is an important operational parameter for the safety, stability and efficiency of the power system. Reliable frequency measurement is prerequisite for effective power control, load shedding, load restoration and system protection. Therefore, there is a need for fast and accurate estimation of the frequency of the power network using voltage waveforms which may be corrupted by noise and harmonics components.

Several digital methods for the frequency measuring have been proposed in the past few decades. The use of the zero crossing detection and calculation of the number of cycles that occur in a predetermined time interval [1] is a simple and well-known methodology. The discrete Fourier transformation, least error squares and Kalman filter are known signal processing techniques, used for the frequency measurement [2-6]. As shown in [7], the bilinear form approach seems to be a very efficient method for both small frequency deviation and offnominal frequency estimation. An adaptive algorithm for frequency measuring over a wide range is suggested in [8]. For generator protection, where frequency is to be estimated over a wide range, an algorithm with a variable window length is proposed in [9]. A Newton-type algorithm has been proposed in [10].

In this paper, a very simple algorithm with acceptable accuracy is derived. The Fourier algorithm is used as digital filter to extract cosine and sine parts of the fundammental frequency component, and the zero crossing technique is applied to cosine and sine parts of the signal. Nonrecursive Fourier algorithm provides cosine and sine components of the fundamental harmonic of the signal. These components are functions of time and have the same frequency as fundamental harmonic of the signal. This fact is well known from the signal processing theory and it is used in this paper. The original signal may be corrupted by noise and then the higher harmonic components. These components may provoke untrue zero crossing points and zero crossing technique would give false frequency of the processing signal.

The cosine and sine components of the processing signal do not contain higher harmonics and have the lower level of noise than the original signal. The zero crossing technique applied to cosine and sine components of the original signal estimates frequency of fundamental harmonic of the processing signal with high accuracy. Therefore, the derived algorithm is very simple and requires modest resources for implementation.

\section{Zero Crossing Technique Applied on Ortogonal Components of Fourier Series}

Let us assume the following observation model of the measured signal $x(t)$ (arbitrary voltage or current), digitized at the measurement location:

$$
x(t)=C \cos (\omega t+\varphi)+R(t)
$$

Signal parameters are: $C$ - magnitude of the fundamental harmonic, $\omega=2 \pi f$ - fundamental harmonic angular 
frequency, $f$ - fundamental harmonic frequency, $\varphi$ phase of the fundamental harmonic, $R(t)$ - part of the signal which contain higher harmonics and zero mean white noise.

If the exact value of $\omega$ is unknown and assumed as $\omega_{a}$, using discrete Fourier series parameter $\mathrm{C}_{a}$ can be estimated using discrete Fourier series as the following:

$$
\begin{aligned}
& \underline{X} \cong \frac{2}{m}\left[\sum_{n=1}^{m} x_{n} \cos \left(\frac{\omega_{a} T_{a}}{m} n\right)-j \sum_{n=1}^{m} x_{n} \sin \left(\frac{\omega_{a} T_{a}}{m}\right)\right]= \\
& =A+j B
\end{aligned}
$$

where: $C_{a}^{2}=A^{2}+B^{2}, \operatorname{tg} \varphi_{f}=\frac{B}{A}, \omega_{a}$ - assumed angular frequency in Fourier series (relation (2) gives exact value of $\mathrm{C}$ only for $\left.\omega_{a}=\omega\right), m$ - number of samples in assumed period of the first harmonic of the processing signal $T_{a}$ $=2 \pi / \omega_{a}, x_{n}-n$-th sample of the signal.

Assumed signal is sampled at frequency $f_{s}=m / T_{a}=1 / T_{s}$, where $T_{S}$ is sampling period. It means that the assumed frequency of the discrete Fourier series is defined by sampling period and number of samples in the assumed period of fundamental harmonic. The cosine and sine components $A$ and $B$ are periodic functions of time. If the frequency of fundamental harmonic of signal is equal to assumed fundamental frequency of Fourier series $\left(T_{s} \cdot m=T_{a}=T=1 / f\right)$, then $A(t)$ and $B(t)$ are ortogonal periodic functions of frequency $f$. If $T_{a} \neq T$ functions $A(t)$ and $B(t)$ are not pure sine and cosine waves but the frequency of their fundamental harmonic is $f$. For the frequency estimation it is enough to observe the cosine, sine or both components of the original signal. The cosine component $A(t)$ is:

$$
A(t)=\sum_{n=1}^{m} x_{n} \frac{2}{m} \cos (\alpha n)
$$

Fig. 1 is shows signal $x$ which contains $100 \%$ the first,
$30 \%$ of the third and $20 \%$ of the fifth harmonic, together with cosine component.

Practical application of the relation (3) requires auxiliary vector of cosine (4) and vector of signal samples (5). The length of the vectors is $m$. The cosine vector is:

$$
\begin{aligned}
& C O=\left[\frac{2}{m} \cos \left(\frac{\omega_{a} T_{a}}{m}\right) \frac{2}{m} \cos \left(2 \frac{\omega_{a} T_{a}}{m}\right) \frac{2}{m} \cos \left(3 \frac{\omega_{a} T_{a}}{m}\right) \ldots\right. \\
& \left.\ldots \frac{2}{m} \cos \left((m-1) \frac{\omega_{a} T_{a}}{m}\right) \frac{2}{m}\right]^{T},
\end{aligned}
$$

The vector of samples is:

$$
S A M=\left[\begin{array}{lllll}
x_{1} & x_{2} & x_{3} & \ldots & x_{m}
\end{array}\right]^{T}
$$

Using auxiliary vector $\mathrm{CO}$ computation in (3) requires only multiplication and addition, without trigonometric function calculation and without division. After the acquisition of a new sample $x_{\text {new }}$ it is necessary to renumber the vector SAM (5) as follows: $x_{1}=x_{2}, x_{2}=x_{3}, \ldots$, $x_{m}=x_{\text {new }}$. Therefore, the moving data window and samples of processing signal are observed as scalars. For each data window relation (3) gives the cosine component $A$.

Thus, the new algorithm for frequency estimation can be now subdivided into the following steps:

1) Signal sample $x_{n}$ acquisition with defined sampling period $\left(T_{s}=\frac{T_{a}}{m}=\frac{1}{m f_{a}}\right)$, where: $T_{a}=1 / f_{a}$ - assumed period of fundamental harmonic (or frequency) of processing signal $\left(T_{a}=0,02 \mathrm{~s}\right.$ for $50 \mathrm{~Hz}$ signal) and $m$ number of samples in assuming period $T_{a}=1 / f_{a}$.

2) Cosine component $A$ calculation using relation (3),

3) Using zero crossing technique it is possible to reckon number of "samples" $m_{\mathrm{A}}$, which contain period of cosine component $A(t)$.

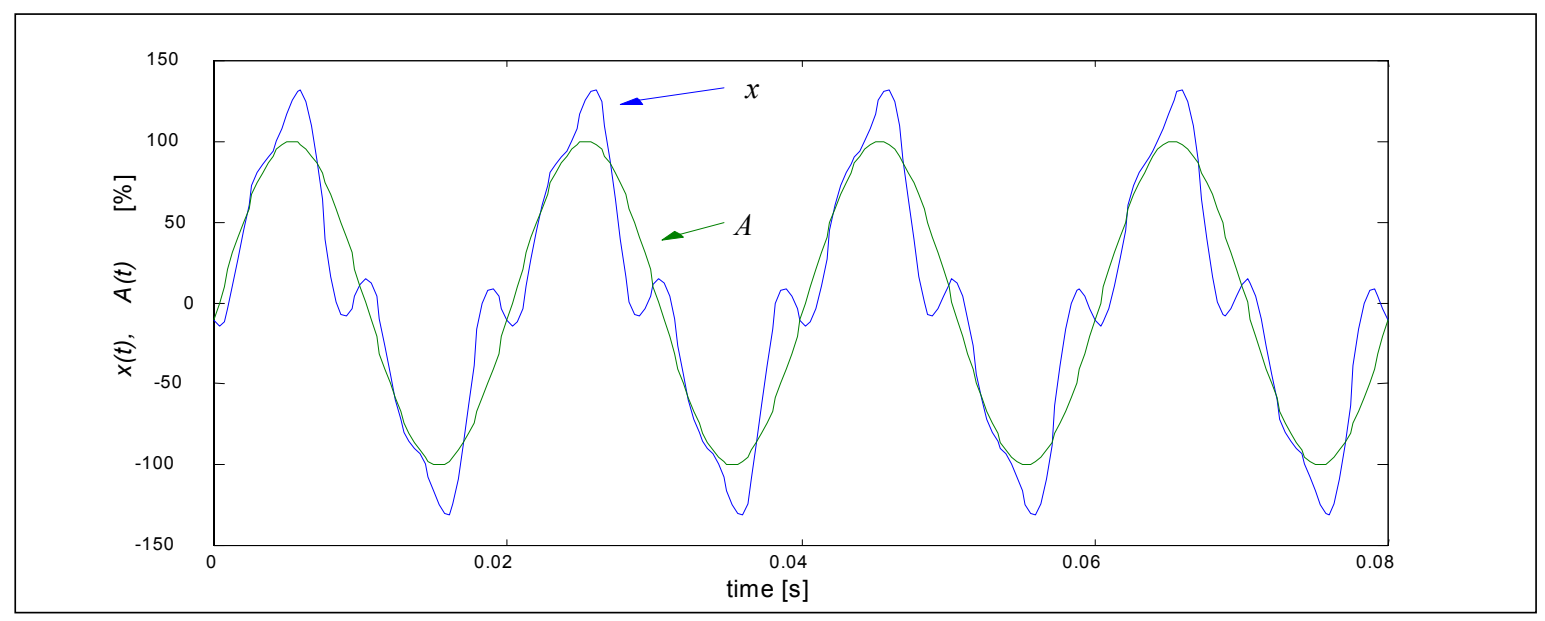

Fig. 1. Original signal $(x)$ and his cosine component (A) 
4) Because of $f_{a}=\frac{1}{m T_{s}}$, the frequency of the fundamental harmonic of processing signal is:

$$
f=\frac{1}{m_{A} T_{s}}=f_{a} \frac{m}{m_{A}} .
$$

Previously defined algorithm has some limitations. When frequencies $f$ and $f_{a}$ are different the period of cosine component $A$ does not contain integer number of samples. For high sampling frequency this fact will cause small error. But for low sampling frequency, the error will be great. Therefore it is necessary to modify previous algorithm. Fig. 2 shows zero crossing of cosine component. of one whole period of the cosine or sine components can be a fraction. After this modification, the algorithm becomes much more accurate. Accuracy of the algorithm also can be enlarged using average number of samples of $M$ periods. One register of length $M$ is needed. In this register numbers of samples of last $M$ periods ( $M$ may be 4 to 10) are stored.

$$
M_{A}=\left[\begin{array}{lllll}
m_{A 1} & m_{A 2} & m_{A 3} & \ldots & m_{A M}
\end{array}\right]^{T}
$$

Register (9) has to be renumbered subsequently to get new number of samples $m_{\text {new }}$. The first sample must be cut off, and the new sample must be added, as follows: $m_{A 1}=m_{A 2}, m_{A 2}=m_{A 3}, \ldots, m_{A M}=m_{\text {Anew }}$.

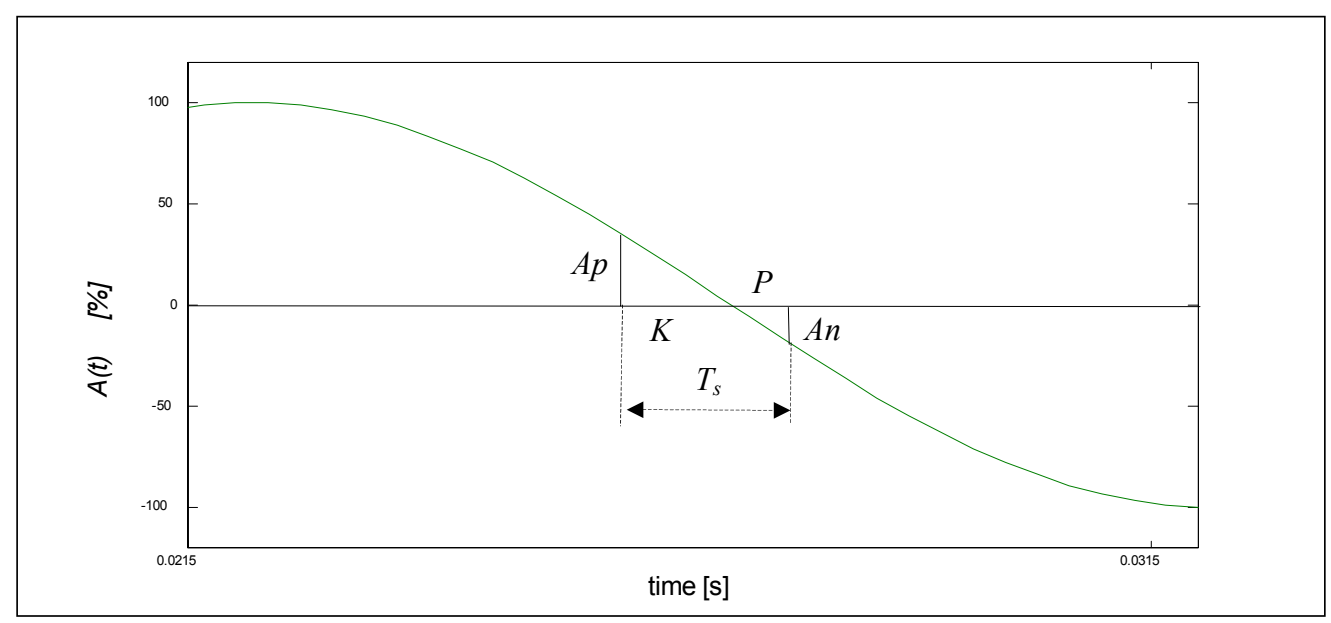

Fig. 2: Zero crossing algorithm correcting

Let us observe the last positive sample $A_{p}$ in previous period and the first sample $A_{n}$ in the next period. In the zero crossing area cosine function can be presented by linear function and the following equations are obtained:

$$
\frac{A_{p}}{K}=-\frac{A_{n}}{P} \quad \text { and } \quad K+P=1
$$

From (7) follows:

$$
P=-\frac{A_{n}}{A_{p}-A_{n}} \quad \text { and } \quad K=\frac{A_{p}}{A_{p}-A_{n}} .
$$

Now we can modify the third step of the previously defined algorithm.

When signal passes through zero, from positive to negative half of period, the number $P(P<1)$ is indicated as the FIRST sample of the following period. At the next signal passing through zero, from positive to negative half of period, the number $K(K<1)$ is indicated as the LAST sample in the previously period and number $(P<1)$ is indicated as the FIRST sample of the next period. All samples between $A_{n}$ and $A_{p}$ which correspond to one period are equal 1 . Sum $P+K$, from the same period can be different from 1 . Therefore, the number of samples $m_{\mathrm{A}}$
The average number of samples of $M$ periods is:

$$
m_{A s}=\frac{m_{A 1}+m_{A 2}+\ldots+m_{A M}}{M},
$$

and the frequency of processing signal is given by:

$$
f=f_{f} \frac{m}{m_{A s}}
$$

This process is the same for each new number of samples of the processing signal period. For practical applications of modified zero crossing technique two registers of length $m$ and one of length $M$ are needed. It means that the presented algorithm requires very small memory resources. It is very simple, accurate and fast.

\section{Performance Evolution Trough Simulation}

The algorithm presented in the previous section is tested by means of the input data obtained through the computer simulations. The static, dynamic and noise tests were performed using computer-simulated test signals. 


\section{A. The Algorithm Sensitivity in the Presence of Harmonics in the Signal}

To demonstrate the effectiveness of the proposed technique for estimating frequency in the presence of harmonics, an input signal having fundamental frequency of $50 \mathrm{~Hz}(100 \%)$, a $30 \%$ third harmonic component and $20 \%$ fifth harmonic component was synthesized. The phases of harmonic components were random functions. Fig.1 shows the waves of synthesized signal. Fundamental frequency in Fourier series was $f_{a}=50 \mathrm{~Hz}$ and the sampling frequency was $f_{s}=1000 \mathrm{~Hz}$. The frequency estimates obtained by zero crossing method applied on original signal and applied on cosine component of original signal are shown in fig.3.

The true values of fundamental frequency of processing signal were obtained, using proposed algorithm, in the sampling frequncy range defined by the Shanon's sampling theorem, i.e., for the $f_{h}<f_{s} / 2=500 \mathrm{~Hz}$, where $f_{h}$ is the highes harmonic frequency in the observed signal. Zero crossing technique applied directly on the signal in presence oh harmonics gives inaccurate estimates of frequency.

\section{B. The Algorithm Sensitivity in the Presence of Noise in the Signal}

The effect of the presence of noise in the signal was studied by estimating the frequency of signals that contain noise. Input sinusoidal $50 \mathrm{~Hz}$ test signal with the superimposed additive white zero-mean Gaussian noise was used in the test. The random noise was selected in order to obtain a prescribed value of the SNR, defined as:

$$
\mathrm{SNR}=20 \log \left(\frac{\mathrm{C}}{\sqrt{2} \sigma}\right)
$$

where $\mathrm{C}$ is the magnitude of the signal fundamental harmonic and $\sigma$ is the noise standard deviation. It should be noted that, in practice, the $S N R$ of voltage signal, obtained from a power system, ranges between (50 and 70) $\mathrm{dB}$. At this level of noise, very small errors are expected with the proposed technique, as depicted in Fig.5. Fig.4 shows the signal corrupted by white noise and his cosine component.

The cosine component is much more close to pure sinusoidal wave than original signal. It can be noted that proposed algorithm has small sensitivity to white noise.

Fig. 5 shows the results of frequency estimation when the signal contanins white Gaussian noise $(\mathrm{SNR}=30 \mathrm{~dB})$. Fig. 5a shows the non averaged estimated frequency and Fig. $5 \mathrm{~b}$ shows the averaged estimated frequency over $M=5$ periods of the signal.

Results shown in Fig. 5 are obtained using fundamental frequency in Fourier series $f_{a}=50 \mathrm{~Hz}$ and sampling frequency $f_{s}=1000 \mathrm{~Hz}$.

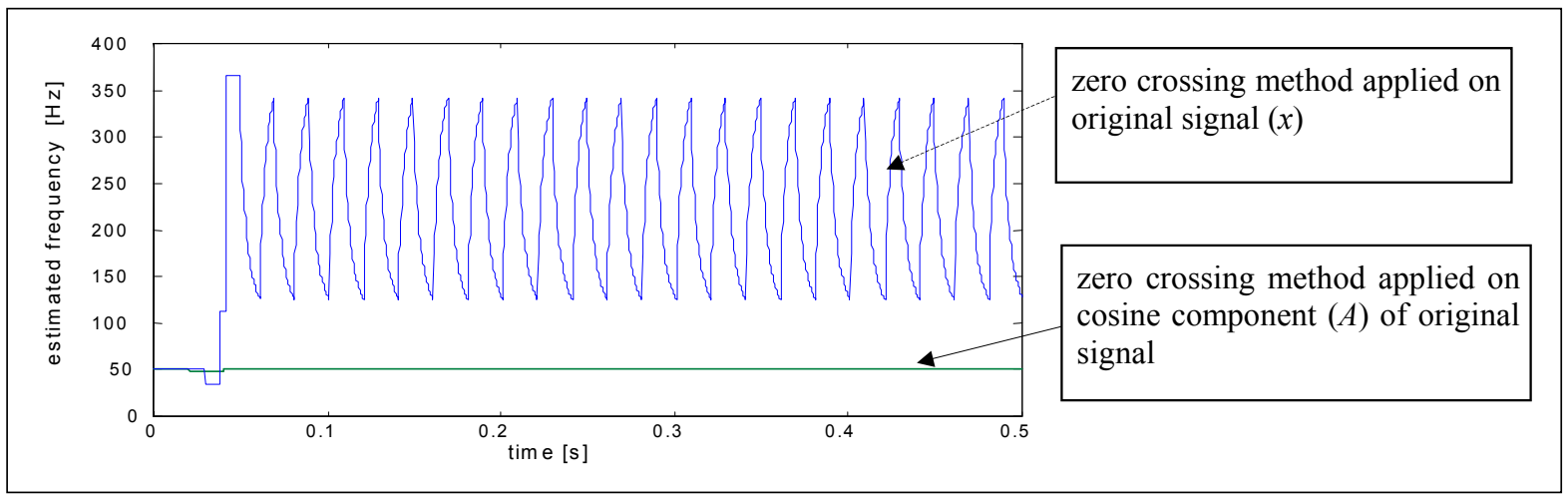

Fig. 3. Frequncy estimates of a $50 \mathrm{~Hz}$ signal shown in fig.1.

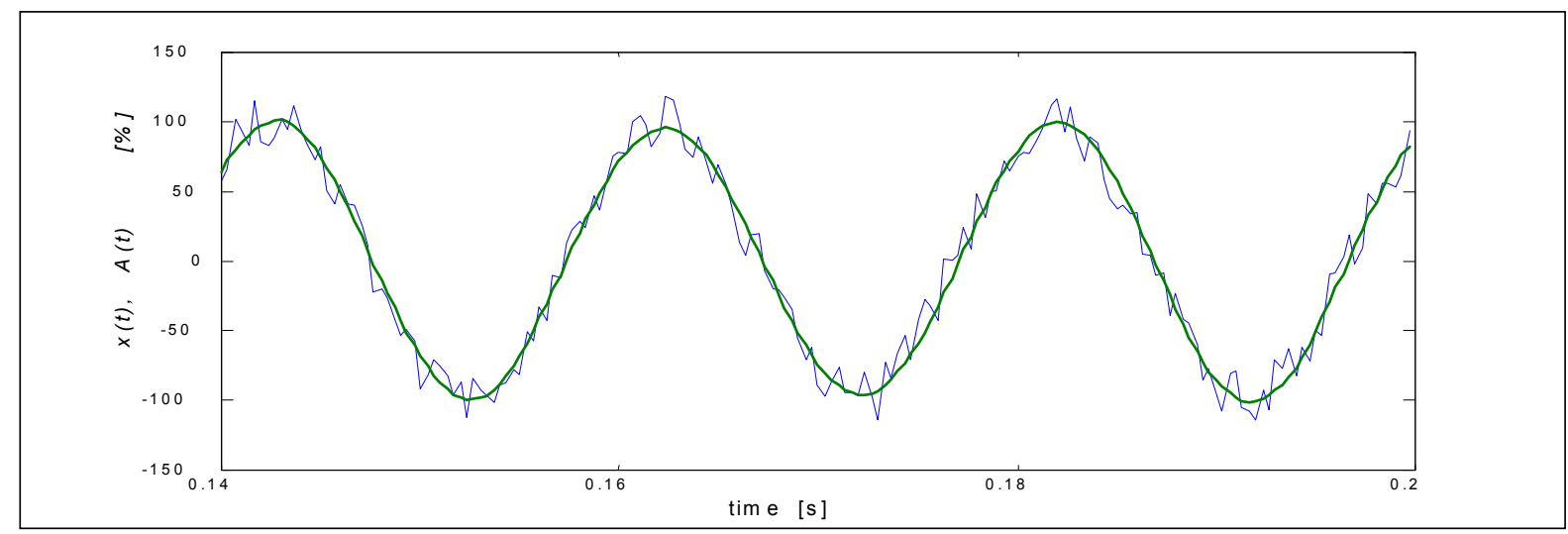

Fig. 4. Test signal $x(t)$ corrupted by noise and his cosine component $A(t)$. 


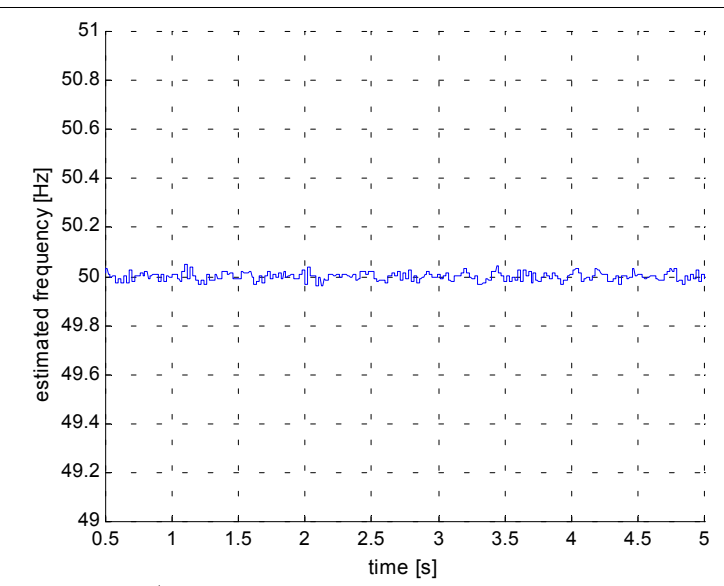

a)

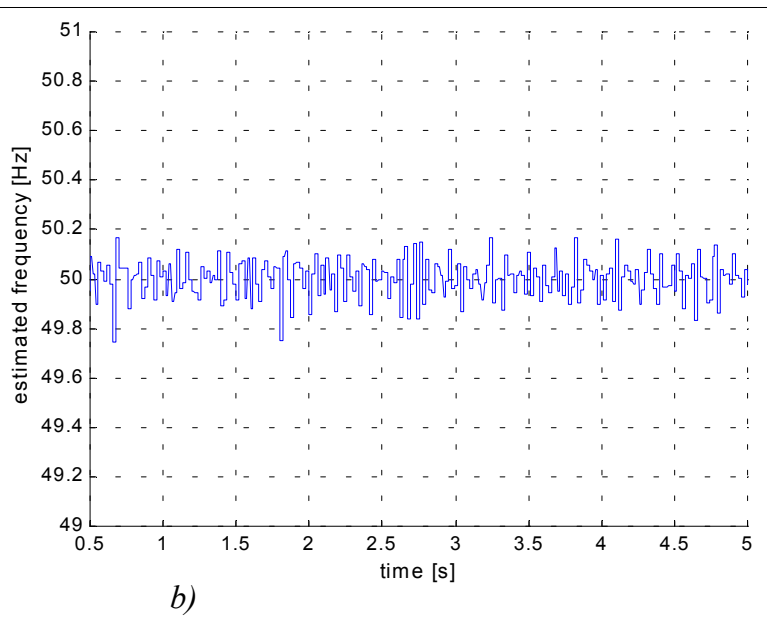

a) non averaged estimated frequency; b) averaged estimated frequency over $M=5$ periods of the signal.

\section{Dynamic Test}

Dynamic behaviour of presented algorithm is tested using two computer simulated signals. In the first test frequency of the sinusoidal signal was changed from $50 \mathrm{~Hz}$ to $55 \mathrm{~Hz}$ and $55 \mathrm{~Hz}$ to $48 \mathrm{~Hz}$ as step function. Fig. $6 \mathrm{a}$ shows results of this test. In the second test magnitude of the sinusoidal $50 \mathrm{~Hz}$ signal was changed from $100 \%$ to $200 \%$ and $200 \%$ to $20 \%$ as step function. Fig. 6 b shows results of this test. In both tests assuming frequency in Fourier series was $f_{a}=50 \mathrm{~Hz}$, and sampling frequency was $f_{s}=1000 \mathrm{~Hz}$.
These dynamic tests show that proposed algorithm is not sensitive to step changes of frequncy and magnitude of processing signal. The algorithm responce to step changes of frequency is delayed because every new value of frequency is the average value of the last 5 periods of the signal.

\section{Real-Life Conditions Test}

To verify presented algorithm, real-life conditions tests are provided. For the first test steady state voltage from distribution network is used. For the second test voltage of single phase motor-generator set is used.

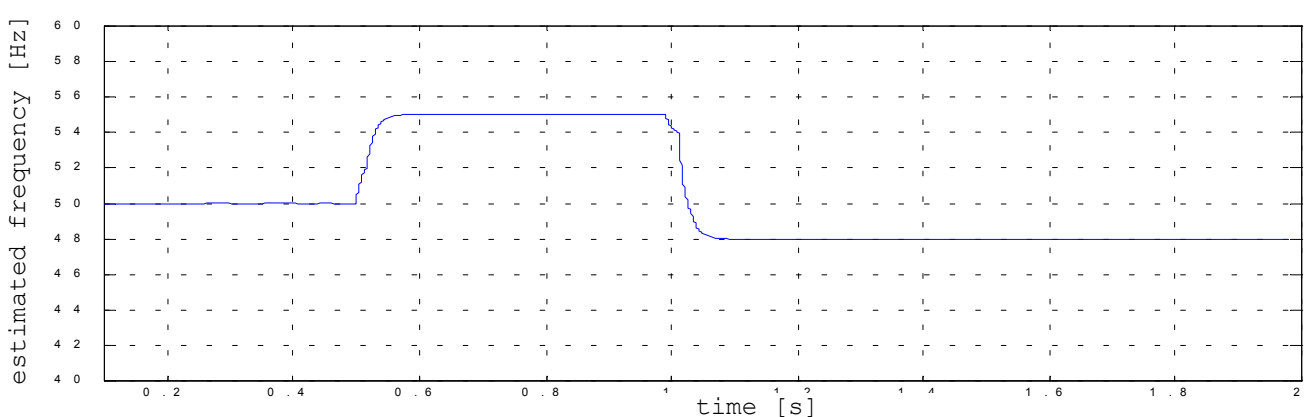

a)

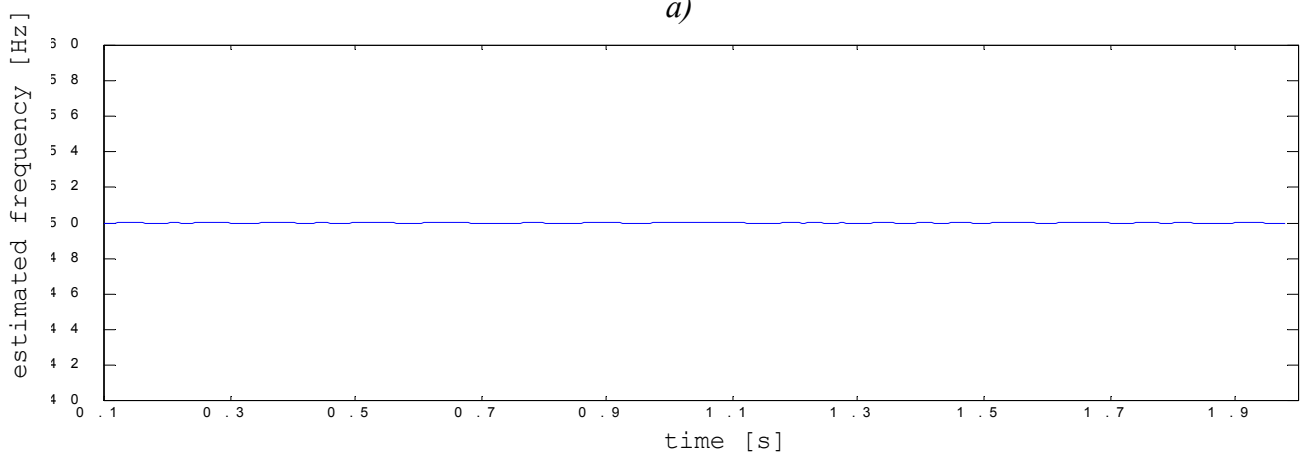

b)

Fig. 6. Frequency estimation; a) when the input signal frequency was step changed and b) when the input signal magnitude was step changed. 


\section{A. Frequency Estimation of Distributing Steady State Network Voltage}

The steady state network voltage samples have been acquired using Data Acquisition Digital System (12-bit A/D converter with sampling frequency $5000 \mathrm{~Hz}$ ) installed at Faculty of Electrical Engineering of Belgrade University. The input signal is shown in Fig.7a. It was corrupted by higher harmonic components. Assuming frequency in Fourier series was $f_{a}=50 \mathrm{~Hz}$. The results of the frequency estimation are given in Fig. $7 \mathrm{~b}$.

To define accuracy of algorithm the actual frequency of the signal was measured by means of Hewleet Packard 5234L Electronic Counter (actual frequency was
$49.877 \mathrm{~Hz})$. The maximum error that occurred in the frequency estimation was less then $0,003 \mathrm{~Hz}$.

\section{B. Frequency Estimation of Motor-Generator Set Voltage}

In this test input voltage is voltage of motor-generator set. This set can run with variable speed. Due to this generator voltage frequency can be function of time, as shown in Fig.8b. Changes of generator voltage magnitude and frequency have caused by step changes of generator load. Generator voltage changes in time are shown in Fig.8a. The results of the frequency estimation are given in Fig. $8 \mathrm{~b}$. The transient test has shown that proposed algorithm has good frequency tracking performance.

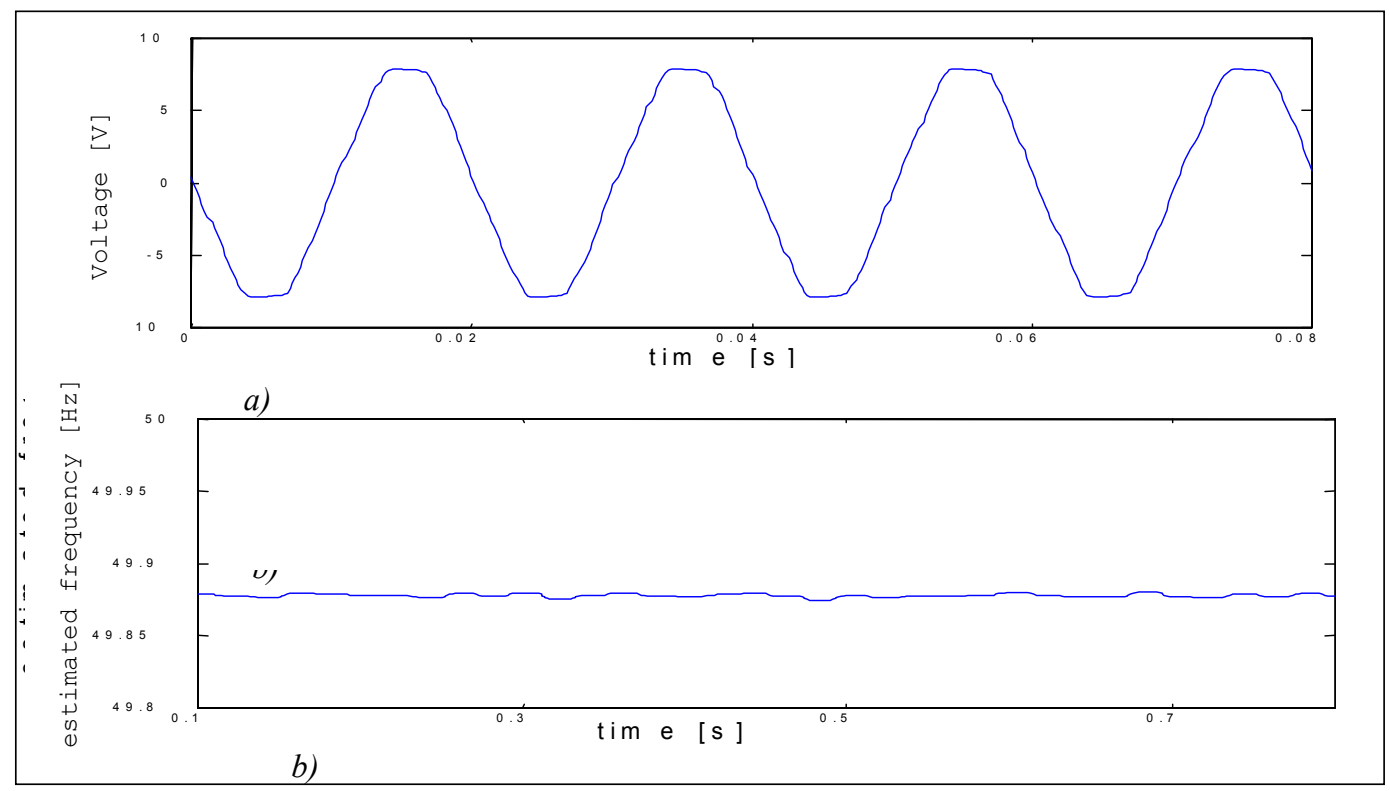

Fig. 7. a) Steady state network voltage waves and b) Frequency estimation.

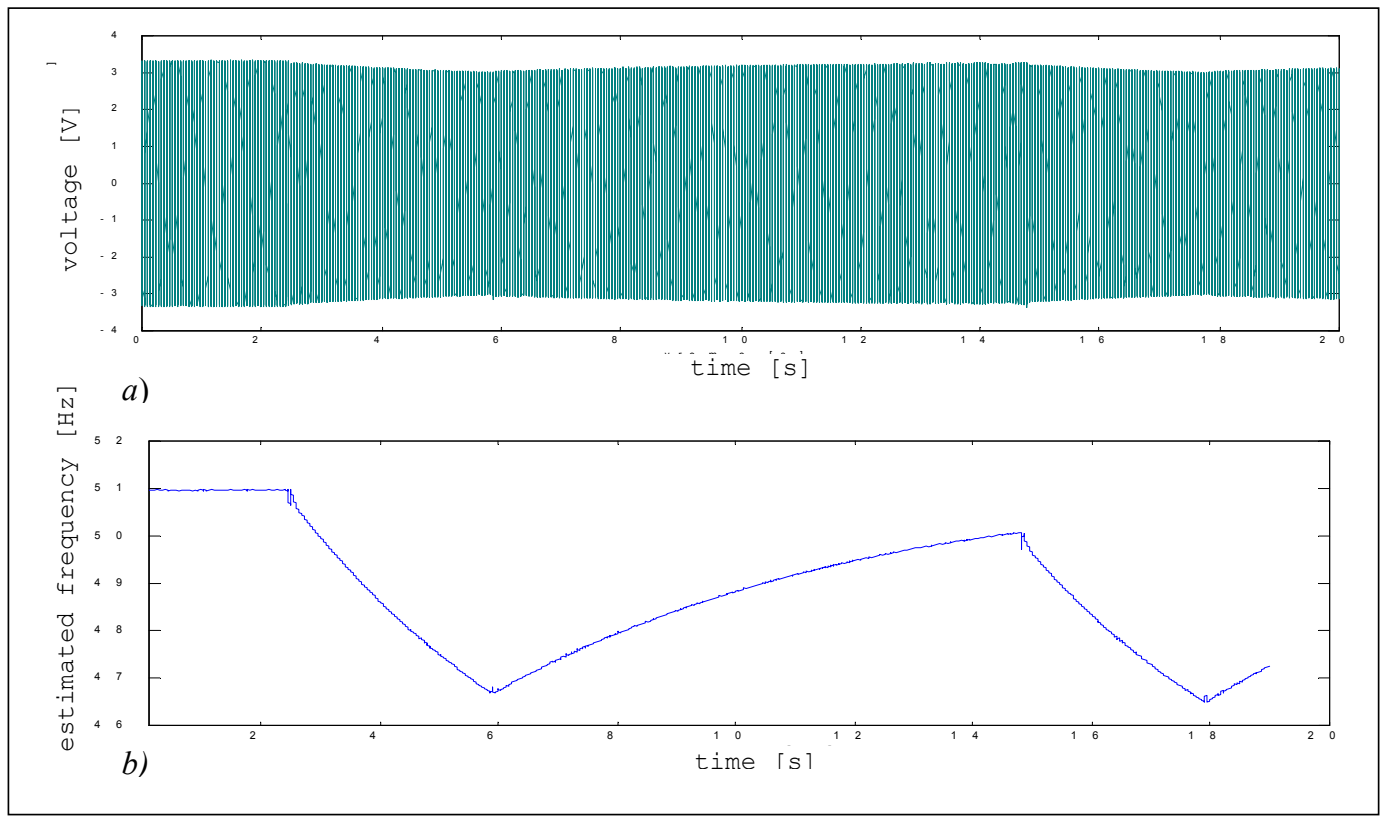

Fig. 8. a) Transient of magnitude of generator voltage and b) Frequency estimation during the voltage magnitude and frequency transient. 


\section{Conclusion}

In this paper a new approach to zero crossing technique for frequency estimation is presented. This approach gives a very simple and robust algorithm with acceptable accuracy. The Fourier algorithm is used as digital filter in order to extract cosine and sine parts of the fundamental frequency component, and the zero crossing technique is applied on cosine and sine parts of the signal. Therefore, the derived algorithm requires modest computer resources for implementation. To verify presented algorithm, computer simulated and real-life conditions tests are provided. All tests have shown that presented algorithn is accurate, wide-range and robust, with good frequency tracking performance. The results of tests confirmed that the algorithm can be a very useful tool in the power system protection applications.

\section{References}

[1] A. Phadke, J. Thorp, and M. Adamiak, "A new measurement technique for tracking voltage phasors, local systems frequency, and rate of change of frequency", IEEE Trans. Power Applicat. Syst., vol. PAS-102, pp. 1025-1038, May 1983.

[2] M. S. Sachdev and M. Nagpal, "A recursive least error squares algorithm for power system relaying and measurement applications", IEEE Trans.Power Delivery, vol. 6, pp. 1008-1015, July 1991.

[3] M. Đurić, Ž. Đurišić, "An Algorithm for OffNominal Frequency Measurements in Electric Power Systems", Electronics, vol. 7, N ${ }^{\circ}$ 1, pp. 1114, Sept. 2003.
[4] A. Girgis and W. Peterson, "Adaptive estimation of power system frequency deviation and its rate of change for calculating sudden power system overloads", IEEE Trans. Power Delivery, vol. 5, pp. 585-594, Apr. 1990.

[5] I. Kamwa and R. Grondin, "Fast adaptive scheme for tracking voltage phasor and local system frequency in power transmission and distribution systems", IEEE Trans. Power Delivery, vol. 7, pp. 789-795, Apr.1992.

[6] V. Eckhardt, P. Hippe, and G. Hosemann, "Dynamic measuring of frequency and frequency oscillations in multiphase power systems," IEEE Trans. Power Delivery, vol. 4, pp. 95-102, Jan. 1989.

[7] M. Kezunović and P. Spasojević, "New digital signal processing algorithms for frequency deviation measurement", IEEE Trans. Power Delivery, vol. 7, pp. 1563-1573, Apr. 1992.

[8] P. J. Moore, R. D. Carranza, and A. T. Johns, "A new numeric technique for high-speed evaluation of power system frequency", Proc. Inst. Elect. Eng., vol. 141, N. 5, pp. 529-536, Sept. 1994.

[9] D. Hart et al., "A new frequency tracking and phasor estimation algorithm for generator protection", IEEE Trans. Power Delivery, vol. 12, $\mathrm{N}^{\mathrm{o}}$. 3, pp. 1064-1073, July 1997.

[10] V. V. Terzija, B. M. Djurić, and B. Kovačcević, "Voltage phasor and local system frequency estimation using Newton type algorithm", IEEE Trans. Power Delivery, vol. 9, pp. 1358-1374, July 1994. 